\title{
IncRNA ROR promotes the proliferation of renal cancer and is negatively associated with favorable prognosis
}

\author{
JIANGUO SHI $^{1,2}$, WEN ZHANG ${ }^{3}$, HONGYANG TIAN $^{2}$, QIANG ZHANG ${ }^{2}$ and TONGYI MEN ${ }^{1}$ \\ ${ }^{1}$ Department of Urology, Qianfoshan Hospital Affiliated to Shandong University, Jinan, Shandong 250014; \\ Departments of ${ }^{2}$ Urology and ${ }^{3}$ Obstetrics, The First Affiliated Hospital of Liaoning Medical University, \\ Jinzhou, Liaoning 121001, P.R. China
}

Received November 2, 2016; Accepted June 15, 2017

DOI: $10.3892 / \mathrm{mmr} .2017 .7775$

\begin{abstract}
Renal cell carcinoma (RCC) is one of the third most common types of urological cancer worldwide. Long non-coding RNA (lncRNA) ROR has been reported to be important in regulating the malignant activities of different types of cancer, however, the function of IncRNA ROR in RCC remains to be fully elucidated. In order to investigate the function of IncRNA ROR in RCC, reverse transcription-quantitative polymerase chain reaction (RT-qPCR) analysis was used to detect the expression of lncRNA ROR in renal cancer tissues and adjacent tissues. Cell proliferation and apoptosis were determined using Cell Counting Kit- 8 and apoptosis assays. Western blot analysis was used to measure the expression levels of c-Myc and p53 following the suppression of IncRNA ROR in RCC cell lines. According to the results of the RT-qPCR analysis, IncRNA ROR was found to be expressed at high levels in RCC tissues and cell lines. Patients with RCC exhibiting high expression levels of IncRNA ROR had shorter survival rates, compared with those with low expression levels of IncRNA ROR. The knockdown of IncRNA ROR resulted in a decrease of cell proliferation and increase of apoptosis in vitro. The suppression of lncRNA ROR also induced an increase in the expression of $\mathrm{p} 53$ and a decrease in the expression of c-Myc in vitro. Taken together, these results demonstrated that lncRNA ROR was expressed at high levels in RCC tissue and cell lines, and was associated with the proliferation ability of RCC cells. These findings indicate that IncRNA ROR may be a promising therapeutic target for treating RCC.
\end{abstract}

\section{Introduction}

Renal cell carcinoma (RCC) is one of the third most common types of urological cancer worldwide (1). According to previous

Correspondence to: Professor Tongyi Men, Department of Urology, Qianfoshan Hospital Affiliated to Shandong University, 16766 Jingshi Road, Jinan, Shandong 250014, P.R. China

E-mail: tongyi_men@yeah.net

Key words: long non-coding RNA ROR, renal cancer, c-Myc, p53, proliferation report, the incidence of RCC has increased in the last 20 years (2\% per year), and patients with advanced RCC experience reduced survival rates $(2,3)$. Chemotherapy, radiotherapy and surgery are the most common treatment methods for RCC, however, response to treatment remains poor (4). In addition, there are few effective and specific biomarkers for RCC, which is the reason for failed early detection and treatment of RCC. As a result, it is necessary to identify sensitive biomarkers for RCC and to develop novel targets for the treatment of RCC.

Previous studies have found that long non-coding RNAs (lncRNAs) are important in the development and progression of cancer $(5,6)$. IncRNAs are a type of non-coding RNA, which are $>200$ nucleotides in length. Certain IncRNAs have been shown to regulate the proliferation, migration and drug resistance of human cancer. Hu et al reported that lncRNA GAPLINC regulates the malignant activity of gastric cancer cells by regulating the expression of CD44 (7). Sun et al found that IncRNA HOXA11-AS promotes the proliferation and invasion of gastric cancer by regulating the histone methylation state and sponging for microRNA (miR)-1297 (8). Yuan et al found that lncRNA DANCR increases the stemness of hepatocellular carcinoma by regulating the expression of CTNNB1 via inhibiting miR-214, miR-320a and miR-199a (9). These findings indicate that lncRNAs may be novel potential targets for treating cancer. However, the function of IncRNAs in RCC remains to be fully elucidated.

lncRNA ROR has been reported as an oncogene in several types of cancer. Studies have shown that lncRNA ROR can promote the proliferation and invasion of breast cancer via targeting miR-145 and miR-205, regulating the targets of these miRNAs $(10,11)$. In addition, previous studies have shown that IncRNA ROR can regulate the sensitivity to radiotherapy and chemotherapy in cancer treatment via the p53/miR-145 signaling pathway, indicating that IncRNA ROR can be used as a novel target in cancer treatment (12). Wang et al also found that lncRNA ROR can predict outcome in patients with gallbladder cancer, and promotes the proliferation, migration and invasion of gallbladder cancer (13). Huang et al found that IncRNA ROR can promote cell proliferation and tumorigenesis by promoting the stability of c-Myc mRNA via binding hnRNP I and AU-rich element RNA-binding protein 1 (AUF1) (14). These studies show that lncRNA ROR is involved in regulating the malignant activities of different types of 
A

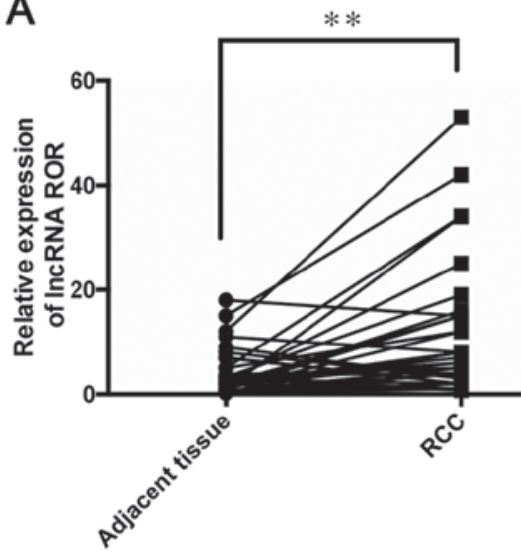

B

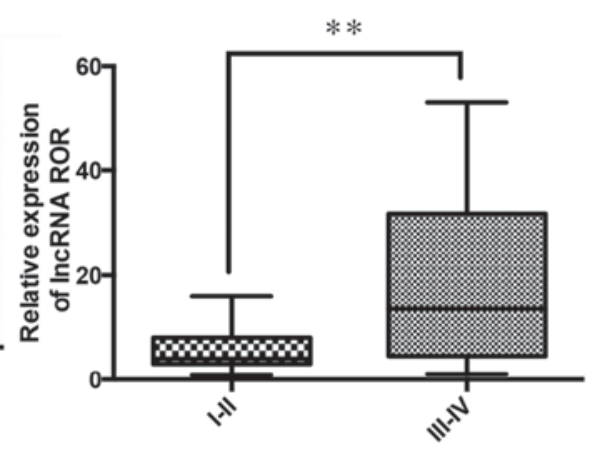

C

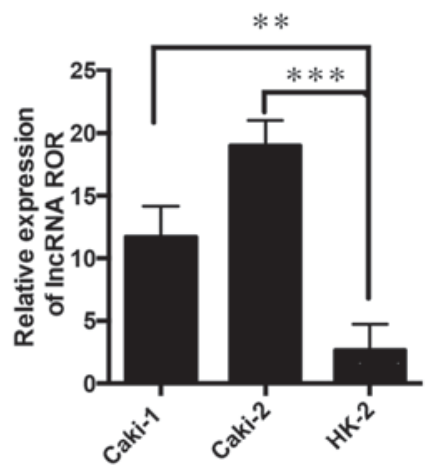

Figure 1. Expression levels of lncRNA ROR are high in RCC tissues and cell lines. (A) Expression levels of lncRNA ROR were detected in 36 patients with RCC using reverse transcription-quantitative polymerase chain reaction analysis. ${ }^{* *} \mathrm{P}<0.01$. (B) Expression levels of lncRNA ROR in clinical stages I and II were compared with levels in clinical stages III and IV. ${ }^{* *} \mathrm{P}<0.01$. (C) Expression levels of lncRNA ROR in RCC cell lines (Caki-1 and Caki-2) and HK-2 cells were measured. ${ }^{* *} \mathrm{P}<0.01 ;{ }^{* * *} \mathrm{P}<0.001$. Unpaired t-tests were used to analyze data. RCC, renal cell carcinoma; lncRNA, long non-coding RNA.

cancer, indicating that IncRNA ROR may be a potential treatment target. However, the role of lncRNA ROR in RCC remains to be elucidated.

The present study aimed to investigate the expression of lncRNA ROR in RCC tissues and adjacent tissues, and to examine the clinical characteristics of lncRNA ROR in RCC. The possible mechanism underlying the effect of IncRNA ROR on RCC was also investigated. The results indicated that IncRNA ROR may offer potential as a novel biomarker for the diagnosis of RCC and a target for its treatment.

\section{Materials and methods}

Clinical sample collection and preparation. A total of $36 \mathrm{RCC}$ tissues and matched adjacent non-tumor tissues were collected from patients who underwent radical nephrectomy in the Department of Urology, The first Affiliated Hospital of Liaoning Medical University (Jinzhou, China), between June 2014 and July 2015. None of these patients had received chemotherapy or radiotherapy prior to collection. The samples were cut into small sections following washing with the RNase-free phosphate-buffered saline (PBS), and then collected and stored in the liquid nitrogen. The clinical stages of the RCC tissue samples were confirmed according to the TNM classification (4). All patients were informed of the use of samples and written consent was provided. The entire protocol was approved by the Institutional Review Board of The First Affiliated Hospital of Liaoning Medical University.

Cell culture. The immortalized normal human proximal tubule epithelial cell line (HK-2) and renal cancer cell lines (Caki-1 and Caki-2) were the cells used for investigation in the present study, which were purchased from the Cell Bank of Type Culture Collection of Chinese Academy of Sciences (Shanghai, China). The HK-2 cell line was cultured in KSFM medium (Hyclone; GE Healthcare Life Sciences, Logan, UT, USA), and Caki-1 and Caki-2 were cultured in RPMI 1640 medium (Hyclone, GE Healthcare Life Sciences). These media were supplemented with 10\% FBS (Gibco; Thermo Fisher
Scientific, Inc., Waltham, MA, USA), and $1 \%$ penicillin and streptomycin (Beyotime Institute of Biotechnology, Haimen, China). The cells were maintained at $37^{\circ} \mathrm{C}$ with $5 \% \mathrm{CO}_{2}$.

Plasmid transfection. The cells were plated in a six-well plate at $70-90 \%$ confluence $\left(\sim 1 \times 10^{5}\right.$ target cells $)$, to which fresh serum-free culture medium was added $2 \mathrm{~h}$ prior to transfection. The plasmids used to knockdown the expression of lncRNA ROR in renal cancer cell lines (shRNA: GATCCC CCCTGAGAGTTGGCATGAATTTCAAGAGAATTCATG CCAACTCTCAGGTTTTTC) were provided by Sangon Biotech (Shanghai, China). The plasmids were transfected into cells using Lipofectamine 2000 (Invitrogen; Thermo Fisher Scientific, Inc.) for $12 \mathrm{~h}$ according to the manufacturer's protocol. The fluorescence was observed after $24 \mathrm{~h}$.

$R N A$ extraction and reverse transcription-quantitative polymerase chain reaction ( $R T-q P C R)$ analysis. Total RNA from the cells and clinical samples were extracted using RNAiso Plus (Takara Bio Inc., Otsu, Japan), according to the manufacturer's protocol. The RNA was then reverse transcribed into cDNA using a PrimeScript ${ }^{\mathrm{TM}}$ RT reagent kit with gDNA Eraser, according to the manufacturer's protocol (Takara Bio, Inc.). The RT-qPCR procedure $(2 \mu \mathrm{l}$ cDNA, $1 \mu \mathrm{l}$ forward primer, $1 \mu \mathrm{l}$ reverse primer, $0.4 \mu \mathrm{l} \mathrm{ROX}, 5.6 \mu \mathrm{l}$ RNase-free Water and $10 \mu \mathrm{l} \mathrm{SYBR}$ ) was performed on a Real time PCR system (Applied Biosystems; Thermo Fisher Scientific, Inc.), as follows: $10 \mathrm{~min}$ at $95^{\circ} \mathrm{C}, 40$ cycles of $10 \mathrm{sec}$ at $95^{\circ} \mathrm{C}, 30 \mathrm{sec}$ at $60^{\circ} \mathrm{C}$, and $20 \mathrm{sec}$ at $72^{\circ} \mathrm{C}$. GAPDH was used as a control. The relative expression of lncRNA ROR (forward: 5'-CCA GGACAATGAAACCAC-3'; reverse: 5'-AGGAGCCCAAAG TAACAG-3') was calculated using the comparative cycle threshold $2^{-\triangle \Delta \mathrm{Cq}}$ method, with GAPDH (forward: 5'-GGT GAAGGTCGGAGTCAACG-3'; reverse: 5'-CAAAGTTGT CATGGATGHACC-3') as the endogenous control for data normalization.

Cell Counting Kit-8 (CCK-8) assays. The target cells were seeded into a 96-well flat-bottomed plate, each well 
Table I. Associations between the expression level of lncRNA ROR and clinical characteristics of patients.

\section{IncRNA ROR}

\begin{tabular}{lcccc}
\cline { 3 - 4 } Characteristic & $\mathrm{n}$ & High (n) & Low (n) & P-value \\
\hline Age (years) & & & & 0.532 \\
$\quad<60$ & 16 & 7 & 9 & \\
$>60$ & 20 & 11 & 9 & \\
Gender & & & & 0.376 \\
$\quad$ Male & 21 & 12 & 9 & \\
Female & 15 & 7 & 8 & \\
Clinical stage & & & & 0.0043 \\
I-II & 19 & 6 & 13 & \\
III-IV & 17 & 13 & 4 & \\
Tumor size & & & & 0.0032 \\
$<3 \mathrm{~cm}$ & 14 & 5 & 9 & \\
$>3 \mathrm{~cm}$ & 22 & 17 & 5 & \\
\hline
\end{tabular}

lncRNA, long non-coding RNA.

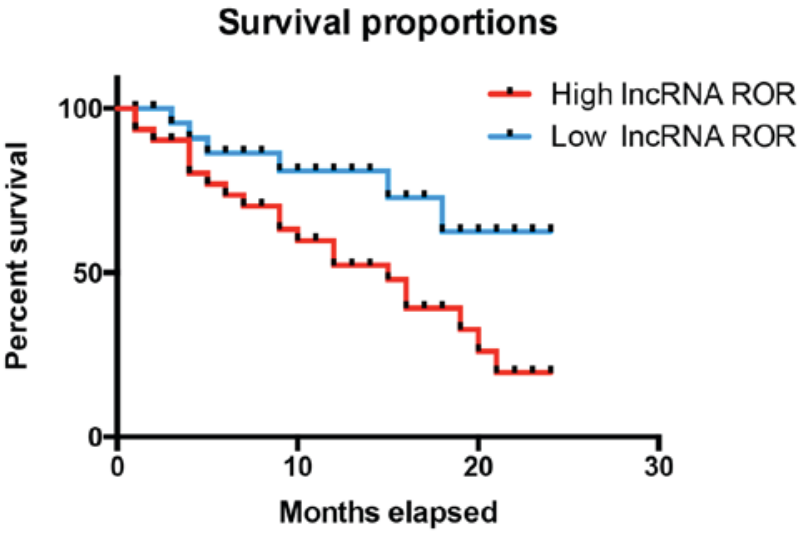

Figure 2. High expression of lncRNA ROR predicts poor prognosis in patients. The association between patient survival rates (log-rank test) and expression of lncRNA ROR was determined $(\mathrm{P}<0.01)$. Survival analysis was performed using the log-rank test in GraphPad software. lncRNA, long non-coding RNA.

containing 3,000 cells in $200 \mu 1$ cell suspension. A $20-\mu 1$ volume of CCK8 (Dojindo Molecular Technologies, Inc., Kumamoto, Japan) was added to each well and cultured for $2 \mathrm{~h}$ at $37^{\circ} \mathrm{C}$. The absorbance value of each well at $450 \mathrm{~nm}$ was measured and recorded, which represented the proliferation of the target cells. Each experiment included five replicates and was repeated three times.

Analysis of apoptosis. The activity of Caspase 3 was measured to determine the level of apoptosis. A Caspase-3 Colorimetric Activity Assay kit (EMD Millipore, Billerica, MA, USA) was used to detect the level of apoptosis of the target cells, according to the standard assay protocol.

Western blot analysis. The cells were washed three times with pre-cooled PBS. Total cell proteins were extracted using RIPA buffer containing the protein inhibitor, PMSF. Protein concentrations were assessed using the standard curve established with bovine serum albumin (Beyotime Institute of Biotechnology, Beijing, China). The total proteins $(30 \mu \mathrm{g})$ were subjected to sodium dodecyl sulfate polyacrylamide gel electrophoresis (SDS-PAGE) on a $10 \%$ denaturing gel. Following SDS-PAGE, the proteins were transferred onto a PVDF membrane (EMD Millipore). The membrane was inhibited with 5\% non-fat milk for $2 \mathrm{~h}$ at room temperature. This was followed by incubation of the membrane with primary antibodies at $4{ }^{\circ} \mathrm{C}$ overnight. The source and dilution of these antibodies were as follows: p53 (ab32049; 1:1,000; Abcam, Cambridge, MA, USA); c-Myc (ab39688; 1:1,000; Abcam); GAPDH (ab8245; 1:1,000; Abcam). The membrane was then washed in TBS-T (Wuhan Boster Biological Technology, Ltd., Wuhan, China) for $5 \mathrm{~min}$, and he membrane was incubated with secondary antibody (A16072; 1:1,000; and A16104; 1:1,000; Thermo Fisher Scientific, Inc.). The blots were visualized using an ECL chemiluminescence detection system, and protein bands were quantified using densitometric analysis with Quantity One software version 4.62 (Bio-Rad Laboratories, Inc., Hercules, USA).

Statistical analysis. All experiments were performed at least three times and data are presented as the mean \pm standard deviation. Statistical analyses were performed using Student's t-test or one-way analysis of variance using GraphPad Prism 5 (GraphPad Software, Inc., La Jolla, CA, USA). Survival analysis was performed using the log-rank test in GraphPad. $\mathrm{P}<0.05$ was considered to indicate a statistically significant difference.

\section{Results}

Expression levels of lncRNA ROR are high in RCC tissues and $R C C$ cell lines. The relative expression of lncRNA ROR was determined in 36 patients with RCC using RT-qPCR analysis. It was found that the expression of lncRNA ROR was high in the RCC tissues, compared with the adjacent non-tumor tissues (Fig. 1A). It was also found that the expression of lncRNA ROR was higher at clinical stages III and IV, compared with that at clinical stages I and II (Fig. 1B). The expression of lncRNA ROR was also detected in Caki-1 and Caki-2 RCC cell lines and the HK-2 normal human proximal tubule epithelial cell line. It was found that the expression levels of lncRNA ROR in the RCC lines were higher, compared with that in the HK-2 cell line (Fig. 1C). These results indicated that lncRNA ROR was expressed at high levels in the RCC tissues and cell lines, and that lncRNA ROR may be involved in the progression and development of RCC.

High expression of IncRNA ROR predicts poor patient prognosis. To further examine the effect of lncRNA ROR in $\mathrm{RCC}$, the present study examined the association between the expression of IncRNA ROR and the survival rates of patients with RCC. It was found that the patients with RCC and high expression levels of IncRNA ROR had reduced survival rates, compared with patients with low expression levels of lncRNA ROR (Fig. 2). As shown in Table I, it was found that the expression of IncRNA ROR was closely associated with tumor size and the clinical stage of RCC, however, no 
A

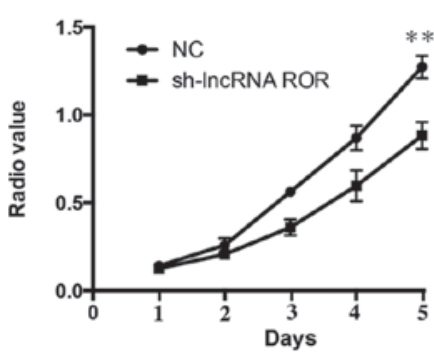

Caki-1
B

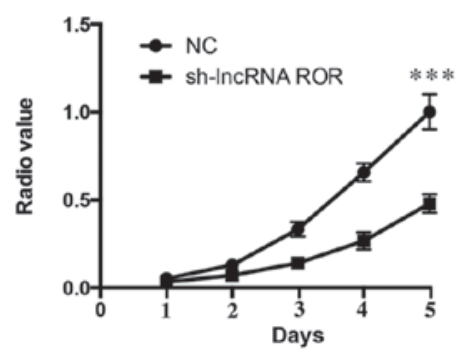

Caki-2

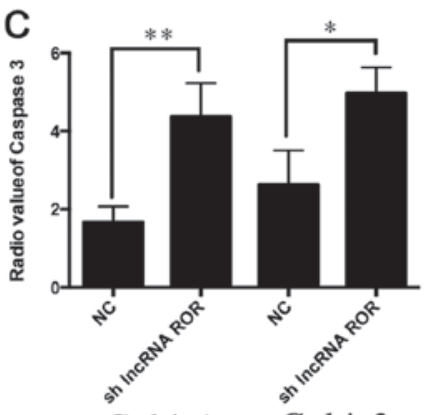

Caki-1 Caki-2

Figure 3. Knockdown lncRNA ROR inhibits proliferation and promotes apoptosis of RCC cells. Proliferation abilities of the (A) Caki-1 and (B) Caki-2 RCC cell lines were detected using a Cell Counting Kit-8 assay following the suppression of lncRNA ROR. ${ }^{* *} \mathrm{P}<0.01 ;{ }^{* * * *} \mathrm{P}<0.001$. (C) Activities of Caspase-3 in RCC cell lines were detected following the suppression of lncRNA ROR. ${ }^{* *} \mathrm{P}<0.01$; ${ }^{*} \mathrm{P}<0.05$. Unpaired t-tests were used to analyze the data. NC, negative control; lncRNA, long non-coding RNA; sh, short hairpin RNA.

A

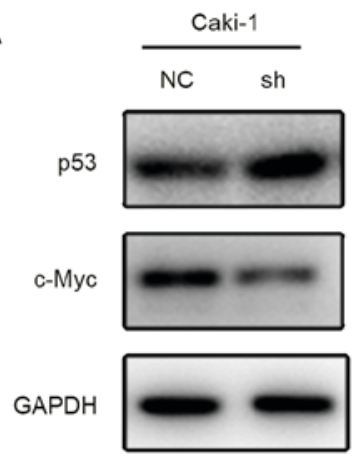

D

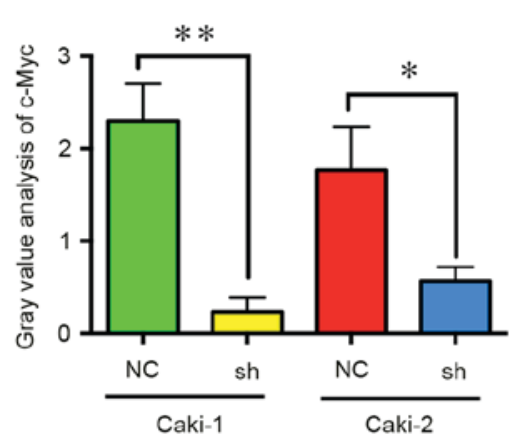

B

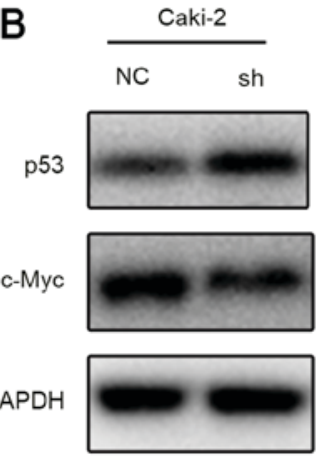

E

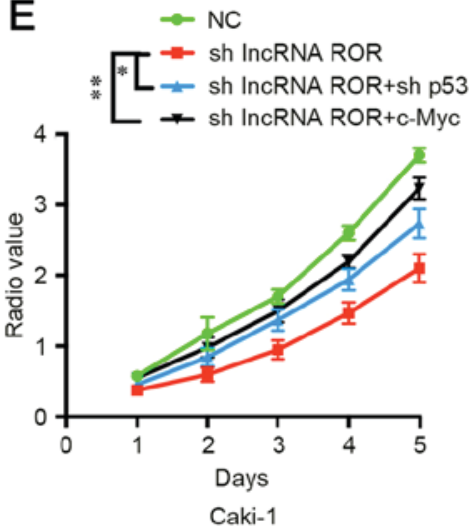

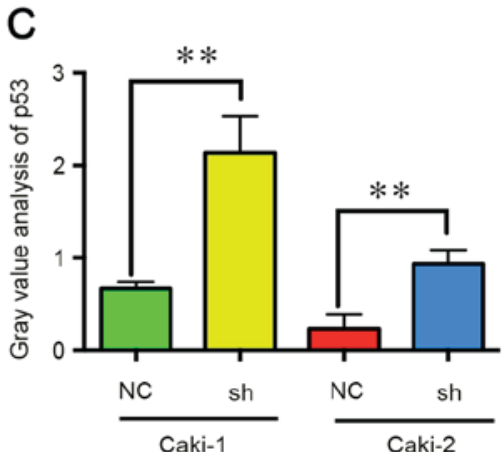

F

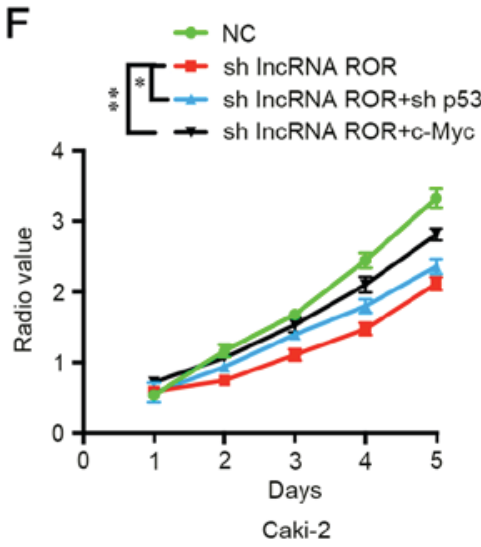

Figure 4. IncRNA ROR promotes the proliferation of RCC via suppressing the expression of p53 and increasing the expression of c-Myc. Expression levels of p53 and c-Myc in (A) Caki-1 and (B) Caki-2 RCC cell lines were detected using western blot analysis. Grey values of (C) p53 and (D) c-Myc in the two RCC cell lines. Proliferation of the (E) Caki-1 and (F) Caki-2 RCC cells were detected using a Cell Counting Kit-8 assay following the suppression of p53 or overexpression of c-Myc. ${ }^{*} \mathrm{P}<0.05 ;{ }^{* *} \mathrm{P}<0.01$. Unpaired t-tests were used to analyze the data. NC, negative control; lncRNA, long non-coding RNA; sh, short hairpin RNA.

significant associations were found between the expression of lncRNA ROR and age or gender. These results indicated that IncRNA ROR was closely associated with tumor size in RCC. Taken together, these results suggested that the expression of IncRNA ROR was closely associated with the prognosis of patients with RCC and may be associated with proliferation.

Knockdown of IncRNA ROR inhibits proliferation and promotes apoptosis of RCC cells. As described in the results above, it was found that lncRNA ROR may be associated with the proliferation of RCC. To further investigate the possible effect of lncRNA ROR on the proliferation of RCC cells, the present study used a CCK8 assay to detect changes in the proliferation ability of Caki-1 and Caki-2 cells following the suppression of IncRNA ROR. It was found that the suppression of IncRNA ROR led to a decrease in the proliferation ability of the Caki-1 and Caki-2 cells (Fig. 3A and B). The apoptosis of Caki-1 and Caki-2 was then detected following suppression of the expression of IncRNA ROR. It was also found that apoptosis was increased following transfection with short hairpin (sh)-lncRNA ROR, compared with that in the control 
group (Fig. 3C). These findings indicated that knockdown of the expression of lncRNA ROR inhibited the proliferation and promoted the apoptosis of RCC cells.

IncRNA ROR promotes the proliferation of RCC cells via suppressing the expression of 553 and increasing the expression of $c-M y c$. Although the present study demonstrated that IncRNA ROR promoted the proliferation of RCC, the detailed mechanism remains to be fully elucidated. According to a previous study, lncRNA ROR promotes the resistance of human colorectal cancer cells to radiotherapy via suppressing the expression of p53. In addition, Huang et al (14) demonstrated that IncRNA ROR promotes the expression of c-Myc to increase cell proliferation and tumorigenesis. In the present study, the expression levels of c-Myc and p53 were detected following the suppression of IncRNA ROR. It was found that the expression of p53 was increased, whereas the expression of c-Myc was decreased (Fig. 4A-D). It was also found that the suppression of p53 and the overexpression of c-Myc restored the proliferation ability of the RCC cells (Fig. 4E and F). These results indicated that IncRNA ROR promoted the expression of c-Myc and suppressed the expression of p53 in the RCC cells, leading to cell proliferation.

\section{Discussion}

RCC remains one of the leading causes of cancer-associated mortality worldwide; therefore, novel targets for its treatment, prognosis and diagnosis are required to improve clinical treatment. An increasing number of studies have found that IncRNA is involved in the development and progression of different types of tumor, which may be developed as novel targets and biomarkers for cancer treatment. Wang et al (15) has found that a panel of four lncRNAs (BANCR, NR_026817, NR_029373 and NR_034119) can be used to detect colorectal cancer. In addition, certain lncRNAs, including lncRNA MALAT1, HOTAIR, IncRNA CCAT2 and H19, have shown potential in predicting prognosis, survival rates and the effect of treatment (16). Among the lncRNAs, IncRNA ROR has been investigated extensively, as its function is important in the development of different types of cancer. However, the effect of lncRNA ROR in the development of RCC has note been fully elucidated, requiring further investigation.

In the present study, the expression of IncRNA ROR in patients with RCC was first investigated. It was found that the expression of IncRNA ROR was high in RCC tissues, compared with that in adjacent non-tumor tissues, determined using RT-qPCR analysis. The results also indicated that the expression of lncRNA ROR was closely associated with patient survival rates. Patients with RCC and higher expression levels of IncRNA ROR experienced reduced survival rates, compared to those with lower expression levels of lncRNA ROR. The expression of lncRNA was also closely associated with tumor size, suggesting that lncRNA ROR affected the proliferation of RCC cells. Taken together, these results indicated that lncRNA $\mathrm{ROR}$ is an independent prognostic factor for patients with $\mathrm{RCC}$, and is important in the proliferation of RCC.

To further confirm the above findings, the effect of lncRNA ROR on the proliferation of RCC cells was determined. Changes in the proliferation ability of the Caki-1 and Caki-2 RCC cells were detected following the suppression of lncRNA ROR. The proliferation of the cells was suppressed, indicating that lncRNA ROR promoted the proliferation of the Caki- 1 and Caki- 2 cells. This result was in agreement with the clinical findings.

Previous studies have found that lncRNA ROR promotes the proliferation of different types of cancer, including the proliferation ability of gastric cancer, gallbladder cancer and nasopharyngeal carcinoma, which indicates that lncRNA ROR is important in controlling the proliferation of cancer $(13,17,18)$. However, the detailed mechanism underlying the effect of lncRNA ROR in regulating proliferation remains to be elucidated. IncRNA ROR was first identified as a regulator for the reprogramming of differentiated cells into induced pluripotent stem cells; the suppression of lncRNA ROR increases apoptosis and the activation of p53 (19). In addition, a previous study demonstrated that lncRNA ROR can act as a competing endogenous RNA to sponge miR-145, and miR-145 is closely linked with p53 (20). Another previous study found that IncRNA ROR also increased the mRNA level of c-Myc in colon cancer and breast cancer cell lines (14). It has also been shown that lncRNA is unable to bind with AUF1, which binds c-Myc mRNA, enhancing the stability of c-Myc mRNA (14). These previous findings suggest that p53 and c-Myc are regulated by lncRNA ROR. According to these findings, the present study aimed to detect whether IncRNA ROR promoted the proliferation ability of RCC via $\mathrm{p} 53$ and c-Myc. The results revealed that the suppression of IncRNA ROR led to an increase in the expression of p53 and a decrease in the expression of c-Myc. It was also found that the overexpression of c-Myc and suppression of p53 restored the proliferation ability of the RCC cells, suggesting that IncRNA ROR may regulate the proliferation ability of RCC cell lines via c-Myc and p53.

In conclusion, the present study found that IncRNA ROR was expressed at high levels in RCC tissues, and its expression was negatively correlated with the survival rates of patients with RCC. This indicated that IncRNA ROR may be used as a potential target to predict the prognosis of RCC. It was also found that lncRNA ROR regulated the proliferation of RCC cell lines, which may occur via regulating the expression of p53 and c-Myc.

\section{References}

1. Siegel R, Naishadham D and Jemal A: Cancer statistics, 2013. CA Cancer J Clin 63: 11-30, 2013.

2. Olshan AF, Kuo TM, Meyer AM, Nielsen ME, Purdue MP and Rathmell WK: Racial difference in histologic subtype of renal cell carcinoma. Cancer Med 2: 744-749, 2013.

3. Torre LA, Bray F, Siegel RL, Ferlay J, Lortet-Tieulent J and Jemal A: Global cancer statistics, 2012. CA Cancer J Clin 65: 87-108, 2015.

4. Russo P: Renal cell carcinoma: Presentation, staging, and surgical treatment. Semin Oncol 27: 160-176, 2000.

5. Esteller M: Non-coding RNAs in human disease. Nat Rev Genet 12: 861-874, 2011.

6. Harries LW: Long non-coding RNAs and human disease. Biochem Soc Trans 40: 902-906, 2012.

7. Hu Y, Wang J, Qian J, Kong X, Tang J, Wang Y, Chen H, Hong J, Zou W, Chen Y, et al: Long noncoding RNA GAPLINC regulates CD44-dependent cell invasiveness and associates with poor prognosis of gastric cancer. Cancer Res 74: 6890-6902, 2014.

8. Sun M, Nie F, Wang Y, Zhang Z, Hou J, He D, Xie M, Xu L, De W, Wang Z and Wang J: LncRNA HOXA11-AS promotes proliferation and invasion of gastric cancer by scaffolding the chromatin modification factors PRC2, LSD1 and DNMT1. Cancer Res 76: 6299-6310, 2016. 
9. Yuan SX, Wang J, Yang F, Tao QF, Zhang J, Wang LL, Yang Y, Liu H, Wang ZG, Xu QG, et al: Long noncoding RNA DANCR increases stemness features of hepatocellular carcinoma by derepression of CTNNB1. Hepatology 63: 499-511, 2016.

10. Hou P,Zhao Y,LiZ, Yao R,MaM,Gao Y,ZhaoL,Zhang Y,Huang B and Lu J: LincRNA-ROR induces epithelial-to-mesenchymal transition and contributes to breast cancer tumorigenesis and metastasis. Cell Death Dis 5: e1287, 2014.

11. Eades G, Wolfson B, Zhang Y, Li Q, Yao Y and Zhou Q: lincRNA-RoR and miR-145 regulate invasion in triple-negative breast cancer via targeting ARF6. Mol Cancer Res 13: 330-338, 2015.

12. Yang P, Yang Y, An W, Xu J, Zhang G, Jie J and Zhang Q: The long noncoding RNA-ROR promotes the resistance of radiotherapy for human colorectal cancer cells by targeting the P53/miR-145 pathway. J Gastroenterol Hepatol 32: 837-845, 2017.

13. Wang SH, Zhang MD, Wu XC, Weng MZ, Zhou D and Quan ZW: Overexpression of LncRNA-ROR predicts a poor outcome in gallbladder cancer patients and promotes the tumor cells proliferation, migration, and invasion. Tumour Biol 37: 12867-12875, 2016.

14. Huang J, Zhang A, Ho TT, Zhang Z, Zhou N, Ding X, Zhang X, Xu M and Mo YY: Linc-RoR promotes c-Myc expression through hnRNP I and AUF1. Nucleic Acids Res 44: 3059-3069, 2016
15. Wang R, Du L, Yang X, Jiang X, Duan W, Yan S, Xie Y, Zhu Y, Wang Q, Wang L, et al: Identification of long noncoding RNAs as potential novel diagnosis and prognosis biomarkers in colorectal cancer. J Cancer Res Clin Oncol 142: 2291-2301, 2016.

16. Xia H, Chen Q, Chen Y, Ge X, Leng W, Tang Q, Ren M, Chen L, Yuan D, Zhang Y, et al: The IncRNA MALAT1 is a novel biomarker for gastric cancer metastasis. Oncotarget 7: 56209-56218, 2016.

17. Wang S, Liu F, Deng J, Cai X, Han J and Liu Q: Long noncoding RNA ROR regulates proliferation, invasion, and stemness of gastric cancer stem cell. Cell Reprogram 18: 319-326, 2016.

18. Li L, Gu M, You B, Shi S, Shan Y, Bao L and You Y: Long non-coding RNA ROR promotes proliferation, migration and chemoresistance of nasopharyngeal carcinoma. Cancer Sci 107: 1215-1222, 2016.

19. Loewer S, Cabili MN, Guttman M, Loh YH, Thomas K, Park IH, Garber M, Curran M, Onder T, Agarwal S, et al: Large intergenic non-coding RNA-RoR modulates reprogramming of human induced pluripotent stem cells. Nat Genet 42: 1113-1117, 2010.

20. Yang P, Yang Y, An W, Xu J, Zhang G, Jie J and Zhang Q: The long non-coding RNA-ROR promotes the resistance of radiotherapy for human colorectal cancer cells by targeting the P53/miR-145 pathway. J Gastroenterol Hepatol 32: 837-845, 2017. 